$\begin{array}{cl}\begin{array}{c}\text { Revue } \\ \text { de } / \text { histoire } \\ \text { des religions }\end{array} & \text { Revue de l'histoire des religions } \\ & \begin{array}{l}\mathbf{4} 2006 \\ \text { Varia }\end{array}\end{array}$

Dionysos mi-cuit : l'étymologie de Mésatis et le festin inachevé des Titans

Half-Boiled Dionysus: The Etymology of Mesatis and the Unfinished Feast of the Titans

Miguel Herrero de Jáuregui

(2) OpenEdition

Journals

Édition électronique

URL : http://journals.openedition.org/rhr/5210

DOI : $10.4000 /$ rhr. 5210

ISSN : 2105-2573

Éditeur

Armand Colin

Édition imprimée

Date de publication : 1 décembre 2006

Pagination : 389-416

ISBN : 978-2-2009-2106-4

ISSN : 0035-1423

Référence électronique

Miguel Herrero de Jáuregui, « Dionysos mi-cuit : l'étymologie de Mésatis et le festin inachevé des

Titans », Revue de l'histoire des religions [En ligne], 4 | 2006, mis en ligne le 28 janvier 2010, consulté le 20 avril 2019. URL : http://journals.openedition.org/rhr/5210 ; DOI : 10.4000/rhr.5210

Tous droits réservés 


\section{Dionysos mi-cuit : l'étymologie de Mésatis et le festin inachevé des Titans}

Selon Pausanias, les savants patréens tiraient le nom de Mésatis, un quartier de la ville, du mythe de Dionysos et des Titans. Ils pensaient probablement au verbe mesazo (être à moitié cuit), puisque Zeus intervient avant que les membres de Dionysos aient eu le temps de cuire complètement. La demi-cuisson de Dionysos est un détail significatif, puisqu'elle exprime l'ambivalence entre le cru et le cuit que l'on retrouve dans d'autres témoignages de ce mythe controversé. Ce détail montre également la perversion titanique du sacrifice traditionnel et, surtout, il révèle la stratégie narrative des citateurs païens et chrétiens du mythe, qui cherchent à éviter de se référer explicitement au tabou de la théophagie.

\section{Half-Boiled Dionysus: The Etymology of Mesatis and the Unfinished Feast of the Titans}

Pausanias tells that the historians of Patras derived the name of the neighbourhood of Mesatis from the myth of Dionysus and the Titans. They were probably thinking of the verb mesazo (to be half-boiled), since Zeus arrived before the Titans had the time to cook Dionysus completely. The half-boiling of Dionysus is a very significant detail, since it expresses the ambiguity between cooked and raw, which is also present in other pieces of evidence for this controversial myth; it is also a detail which shows the Titanic perversion of the normal sacrifice; and above all, it reveals the narrative strategy of the narrators of this myth, both pagans and Christians, to avoid making explicit mention of the taboo of theophagy. 


\section{UNE ÉTYMOLOGIE NÉGLIGÉE}

Dans le $7^{\mathrm{e}}$ livre de sa Périegèse, dédié à la région d'Achaïe, Pausanias relate l'histoire de Patras (7, 18, 2-7). Ses sources, nous dit-il, sont «les historiens des plus hautes antiquités de Patras». Bien que l'on ne puisse totalement exclure une information de type oral, le Périégète se réfère sans doute aux chroniques écrites des historiens locaux ${ }^{1}$. Quoi qu'il en soit, Patras semble s'être formée par la fusion de trois villes, Aroé, Anthéia et Mésatis². Pausanias commence par raconter la fondation des deux premières villes par l'autochtone Eumèlos aidé de Triptolème, puis il en donne l'étymologie : Aroé rappelle le travail de la terre (ơoó $\omega)$ et Anthéia le nom d'Anthéias, fils d'Eumèlos, mort de sa chute du char de Triptolème. Pausanias ajoute ensuite $(7,18,4)$ :

Une troisième cité fut fondée à son tour entre Anthéia et Aroé, il s'agit de Mésatis (Mعбótıc). Quant à tout ce que disent les Patréens au sujet de Dionysos, qu'il fut élevé à Mésatis et que là, victime d'un complot

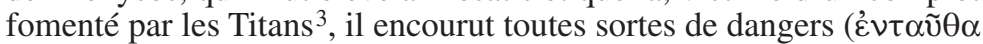

1. Il utilise la même expression pour désigner ses sources éléennes $(5,7$, $6)$, arcadiennes $(8,6,1)$ eubéennes $(8,12,14)$, péloponnésiennes $(8,34,4)$. Il s'agit dans tous les cas d'épisodes mythiques en lien avec le lieu, comme dans le cas de Patras, et de débats chronologiques dont le caractère livresque semble incontestable. La réhabilitation actuelle de Pausanias comme source pour l'histoire de la Grèce d'époque impériale doit beaucoup aux stimulants travaux de Christian Habicht, Pausanias' Guide to Ancient Greece, Berkeley-Los AngelesLondres, 1985.

2. Athanasios D. Rizakis, Achaïe I : Sources textuelles et histoire régionale, Athènes 1995, 164s essaie de localiser Mésatis dans la zone de Voudéni (ou Skioessa)-Anô Sychaina, 4, $5 \mathrm{~km}$ au nord-ouest, où l'on trouve des traces de présence continue depuis l'époque mycénienne jusqu'à l'époque paléochrétienne, à l'exception de la période géométrique. Il semble qu'il s'agissait d'une zone viticole (Jules Herbillon, Les cultes de Patras avec une prospographie patréene, Baltimore-Londres 1929, 122), ce qui explique le culte de Dionysos Mésateus abordé ci-dessous.

3. Les manuscrits transmettent $\pi \alpha \nu \tilde{\omega} \nu(\mathrm{VF})$ et $\pi \alpha ́ \nu \omega \nu(\mathrm{P})$. Mais la correction de Clavier Tıtó $\nu \omega \nu$ est généralement acceptée : on ne trouve aucune source faisant de Pan, a fortiori de plusieurs Pans, les assassins de Dionysos, contrairement à ce qu'il en est des Titans. En revanche, ils sont toujours, comme les silènes ou les satyres, du côté de Dionysos. En outre sur le plan paléographique, les lettres TIT peuvent être facilement confondues avec un $\Pi$. 


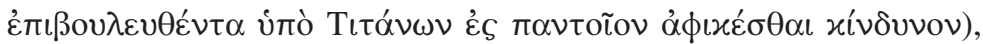
je ne veux pas contredire les gens de Patras et leur laisse à eux-mêmes le soin d'expliquer le nom de Mésatis (trad. Lafond).

Il explique ensuite comment, après avoir expulsé les Ioniens, Patrée, chef des Achéens, fonda Patras à partir des trois villes. Pausanias ne fournit aucun détail supplémentaire sur l'étymologie que les érudits patréens donnent de ce qui était alors un quartier périphérique de Patras. On peut expliquer ce silence par le scepticisme de notre auteur. Avec une courtoisie toute ironique, il préfère ne pas révéler une étymologie qui viendrait contredire celle qui

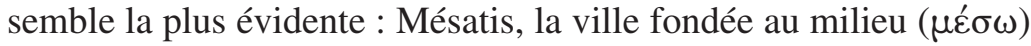
des deux autres. On a aussi suggéré que ce silence s'est imposé à Pausanias par sa réserve devant un mythe étroitement lié au monde des mystères, à l'instar de la discrétion dont fait preuve son modèle Hérodote devant les hieroi logoi ${ }^{4}$. En effet, le mythe du meurtre de l'enfant Dionysos par les Titans, qui le démembrent, le cuisinent et le mangent (trois faits auxquels Pausanias fait allusion par l'euphémisme « toutes sortes de dangers »), est fermement lié à la tradition littéraire et rituelle orphique, dont il est souvent considéré comme la clé anthropologique ${ }^{5}$. Peut-être le silence de Pausanias n'est-il

4. Herbillon, Cultes de Patras, 120-122. Cf. p. ex. Hdt. 2, 13, 4 et 8, 15, 4. Sur ce sujet, D. Foccardi, "Religious Silence and Reticence in Pausanias", dans M. G. Ciani (éd.), The Regions of Silence: Studies on the Difficulty of Communicating, Amsterdam 1987, 67-113, et Albert Henrichs, "Hierai Bibloi and Hieroi Logoi: The (Un)written Margins of the Sacred in Ancient Greece", Harvard Studies in Classical Philology, 101 (2003), 207-266 (spéc. nn. 102 et 143 ).

5. Alberto Bernabé : «La toile de Pénélope : a-t-il existé un mythe sur Dionysos et les Titans ? », Revue de l'histoire des religions, 219 (2002), 401-433, et «Autour du mythe orphique sur Dionysos et les Titans : quelques notes critiques », dans D. Accorinti et P. Chuvin (éds.), Des Géants à Dionysos : mélanges de mythologie et de poésie grecques offerts à Francis Vian, Alessandria 2003, 25-39, fait une réfutation convaincante des dernières positions sceptiques qui ne croient pas possible une interprétation anthropologique ancienne du mythe, notamment Luc Brisson, « Le corps dionysiaque : l'anthropogonie décrite dans le Commentaire sur le Phédon de Platon attribué à Olympiodore est-elle orphique ? », Orphée et l'Orphisme dans l'Antiquité gréco-romaine, Aldershot 1995, 481-499, et Radcliffe G. Edmonds, "Tearing Apart the Zagreus Myth : A Few Disparaging Remarks on Orphism and Original Sin", Classical Antiquity 18 (1999), 35-73. 
pas seulement motivé par sa condescendance envers les sages patréens, mais aussi par le respect que le sujet lui inspire: dans une autre allusion au meurtre de Dionysos $(8,37,5)$, il se sert également d'un euphémisme, «souffrances » $(\pi \alpha \theta \dot{n} \mu \alpha \tau \alpha)$. Quoi qu'il en soit, les érudits locaux tiraient le nom de Mésatis de l'épisode des Titans, afin de mettre en évidence qu'il s'était produit précisément à cet endroit

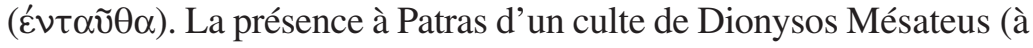
côté de ceux consacrés à Dionysos Aroeus et Antheus : 7, 21,6) explique le désir d'associer la ville à son nom par une étymologie artificielle, typique des historiens locaux. Les érudits modernes semblent cependant avoir renoncé à rechercher cette étymologie ${ }^{6}$.

Il me semble toutefois possible de proposer l'explication du mot que les Patréens auraient pu envisager. Les hypothèses sont assez peu nombreuses, puisqu'il paraît assuré que seul un mot commençant également par $\mu \varepsilon \sigma$ - puisse être accepté comme étymologie populaire du nom de Mésatis, étant donné la proximité phonétique des noms d'Aroé et Anthéia avec leur étymologie correspondante. Parmi le peu de candidats figurant au dictionnaire, celui qui se prête le mieux à cette explication est le verbe $\mu \varepsilon \sigma \alpha ́ \zeta \omega$, dont les quatre premières lettres coïncident parfaitement avec le nom de la ville (avec un $\alpha$ bref comme dans Naucratis) et dont le suffixe dental présente une similarité évidente avec - $\tau \iota s$. Ce verbe est par ailleurs le seul mot que l'on peut mettre en relation avec le mythe de Dionysos et des Titans, une relation dont il est possible de tirer quelques conclusions intéressantes.

Cela étant dit, il faut préciser d'emblée qu'aucune des réflexions présentées ci-dessous ne dépend de l'hypothèse selon laquelle $\mu \varepsilon \sigma \alpha ́ \zeta \omega$ était l'étymologie populaire de Mésatis. Celle-ci me paraît vraisemblable, mais non démontrable. Si le passage de Pausanias a été retenu comme point de départ de cette étude c'est parce qu'il permet d'attirer l'attention sur un détail significatif ignoré jusqu'ici, à savoir que, dans certaines versions du mythe, Dionysos n'a été cuit qu'à moitié.

6. Herbillon, Cultes de Patras, 120 : «Les Patréens proposaient une autre étymologie qu'il faut renoncer à deviner ». Les recherches postérieures sur l'histoire de Patras (Rizakis, Achaïe) ne s'y attellent pas non plus. La seule proposition que je connaisse est celle de Robertson, sur laquelle je reviendrai ci-dessous ( $c f$. n. 7). 


\section{LA CUISSON IMPARFAITE DE L'ENFANT DiONYSOS}

Le verbe $\mu \varepsilon \sigma \alpha ́ \zeta \omega$ signifie d'abord et avant tout «être au milieu ». On pourrait penser que Dionysos est au milieu des Titans qui le démembrent, mais l'étymologie serait alors bien banale ${ }^{7}$. Il semble plus indiqué de prendre ici le verbe dans son acception culinaire : «être à moitié cuit ${ }^{8}$. En effet, dans les récits les plus détaillés du mythe, il semble que les membres de Dionysos n'ont pas eu le temps de cuire complètement, puisque Zeus surprend les Titans avant qu'ils n'aient le temps d'achever leur festin. Quand Clément d'Alexandrie relate cet épisode ${ }^{9}$, il passe immédiatement du moment où les membres de Dionysos sont mis au feu à l'arrivée soudaine de Zeus :

Les Titans, qui l'avaient démembré ( $\delta \iota \alpha \sigma \pi \alpha ́ \sigma \alpha \nu \tau \varepsilon \varsigma$ ), plaçant une marmite sur un trépied, y jetèrent ses membres ; d'abord ils les firent bouillir, ensuite ils les transpercèrent avec de petites broches et « les

7. C'est la suggestion de Noel Robertson, "Orphic Mysteries and Dionysiac Ritual”, dans M. B. Cosmopoulos (éd.), The Greek Mysteries, Londres 2003, 236 n. 45 : «Pausanias emphasizes the looming threat which the Titans posed,

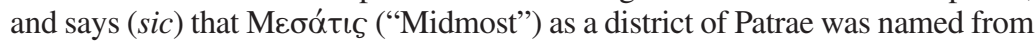
the episode, presumably because the child was surrounded by his enemies. »

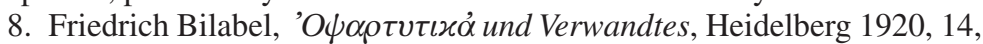
met en évidence deux cas d'utilisation du mot dans un papyrus qui contient des recettes de cuisine, avec un sens identique à Apicius 153: cum mediaverint coctura. Le papyrus date probablement du $\mathrm{III}^{\mathrm{e}}-\mathrm{IV}^{\mathrm{e}} \mathrm{s}$. de notre ère, mais Bilabel pense qu'il peut transmettre des recettes de Mithaikos (antérieur au $\mathrm{IV}^{\mathrm{e}} \mathrm{s}$. av. J.-C.) ou Epainétos (antérieur au I ${ }^{\mathrm{er}}$ s. av. J.-C.). Dans Aetius, Liberia médicinales 3, 61, 9, le verbe peut avoir aussi un sens culinaire. Ce sens est clairement dérivé de l'usage le plus commun du verbe, «être à moitié du temps », qu'on dit par exemple du jour ou de la nuit (cf. LSJ s. v.).

9. Protr. 2, 18, 1. La source de Clément pour sa description des mystères grecs (Protr. 2.12.22) est un traité sous forme alphabétique sur les mystères grecs d'époque hellénistique (Christoph Riedweg, Mysterienterminologie bei Plato, Philo und Klemens von Alexandrien, Berlin-New York 1987, 117-123) qui, à son tour, s'inspire d'une théogonie orphique plus ancienne : outre les épisodes de Dionysos et Baubo qui sont illustrés par des vers d'Orphée, les incestes de Zeus avec Déméter et Perséphone sont propres à la tradition orphique ; l'apparition des jouets de Dionysos (OF 316 Bernabé) dans le P. Gurôb (OF 578 B) de 275 avant J.-C. permet de faire remonter cette théogonie orphique au début de l'époque hellénistique. $C f$. mon article, «Las fuentes de Clem. Alex. Protr. 2.12-22: un tratado sobre los misterios y una teogonía órfica », Emerita : Revista de lingüística y filología clásica (à paraître). 
tinrent au-dessus d'Héphaïstos » (Il. 2.426-428). Et enfin Zeus apparaît... (Traduction de Mondésert légèrement modifiée)

Arnobe, qui fait une traduction libre de Clément en apportant des détails nouveaux à sa description des mystères grecs ${ }^{10}$, souligne la rapidité et le caractère inattendu de l'arrivée de Jupiter, attiré au moment même où l'odeur de la cuisson se fait sentir :

On renonce à expliquer les autres Bacchanales... et que Bacchos, étant occupé à des jeux puérils, a été dépecé par les Titans et découpé membre après membre par eux, puis jeté dans des marmites pour qu'il cuise ( $a b$ isdem membratim sectus atque in ollulas coniectus ut coqueretur), de même qu'on renonce à expliquer comment Jupiter, séduit par le délice de l'odeur, est arrivé rapidement au repas sans être invité (suavitate odoris inlectus, invocatus advolarit ad prandium) et, ayant compris l'affaire, a détruit les brigands avec une terrible foudre et les a envoyés dans les demeures les plus profondes du Tartare (ma traduction).

On rencontre chez Nonnos un saut narratif encore plus grand. Non seulement il ne précise pas, malgré son goût pour les détails les plus baroques des mythes, que Dionysos a été dévoré, mais il évite même de décrire sa mise au feu ${ }^{11}$ :

À tour de rôle, à coups de coutelas, ses meurtriers découpent menu Dionysos sous les espèces d'un taureau.

Mais lorsque Zeus le Père s'aperçoit qu'une forme ombreuse, au piège d'un miroir, a causé le démembrement du premier Dionysos, après avoir frappé la mère des Titans d'une flamme vengeresse il enferme les meurtriers de Zagreus cornu derrière les portes du Tartare. (trad. Chuvin)

On dirait que l'arrivée soudaine de Zeus ne permet pas de cuire totalement les membres de Dionysos puisque, dans ces trois textes

10. Adv. nat. 5, 19. Fabio Mora, Arnobio e i culti de mistero, Roma, 1994 essaie de montrer qu'Arnobe se serait inspiré principalement d'une source différente de Clément, bien qu'il doive admettre que l'apologiste africain a connu le Protreptique. Mais les concordances entre eux sont trop frappantes, à mon avis, pour rejeter la thèse traditionnelle avancée par Emanuele Rapisarda, Clemente Fonte di Arnobio, Turin, 1939.

11. Dion. 6, 204-210. Dans l'introduction au troisième tome de son édition de Nonnos dans Les Belles Lettres (collection Budé), Chuvin fait l'observation suivante : «Proclus et Nonnos se contentent de dire que la victime a été découpée... peut-être éprouvent-ils, pour mentionner ce cannibalisme, un scrupule étranger à Firmicus Maternus, ardent converti au christianisme ? » Nous essaierons de répondre à cette question au point 5 de cet article. 
au moins, les Titans ne semblent même pas avoir le temps de se préparer à manger. Dionysos serait alors cuit à moitié. Avant d'analyser les implications de ce fait, soulignons que la demi-cuisson semble être l'expression d'une ambiguité entre le cru et le cuit que l'on retrouve dans trois témoignages clairement liés au mythe des Titans :

1. Dans le P. Gurôb, qui conserve des instructions pour une célébration rituelle du mythe de Dionysos, on lit (lignes 2-3), «le rassemblement de viande crue... pour la téleté » et, peu après (ligne 15), très probablement, « offrir ce qui est consommé » ${ }^{12}$.

2. Le chrétien Firmicus Maternus, dans une version évhémériste du mythe sur laquelle nous reviendrons, dit que les membres de Dionysos ont été « cuits de différentes façons » (decocta variis generibus), et il ajoute peu après que, pour commémorer l'événement, les Crétois « rééditent point par point tout ce que l'enfant a fait ou souffert en mourant. Ils déchirent à belles dents un taureau vivant...» (omnia per ordinem facientes quae puer moriens aut fecit aut passus est. Vivum laniant dentibus taurum...). La contradiction est évidente ${ }^{13}$.

3. Dans les fragments de papyrus qui nous sont conservés du roman Phoinikika de Lollianos, dont quelques scènes s'inspirent de rituels mystériques (mythiques ou réels) ${ }^{14}$, se lit le récit d'un festin

12. Le $P$. Gurôb $(O F 578 \mathrm{~B})$, daté environ de 275 avant J.-C, a été réédité et commenté récemment par James H. Hordern, "Notes on the Orphic Papyrus from Gurôb (P. Gurôb 1; Pack ${ }^{2}$ 2464)", Zeitschrift für Papyrologie und Epigraphik, 2000 (129), 131-140.

13. De err. 6, 3-5 (trad. Turcan). Cette mention de l'omophagie en contradiction avec une autre proposition juxtaposée s'apparente à un autre texte de tonalité orphique : les ascètes du cœur des Crétois d'Euripide, qui disent pratiquer l'omophagie en même temps que le végétarisme (Fr. 472 Kannicht =OF 567 B). Sur les sources de Firmicus, $c f$. n. 32 infra.

14. Albert Henrichs, Die Phoinikika des Lollianos, Bonn 1972 ; Susan A. Stephens, John J. Winkler, Ancient Greek Novels: The Fragments, Princeton 1995, 314-357. J. Winkler, "Lollianos and the Desperadoes", Journal of Hellenic Studies, 100 (1980), 155-181, montre que les scènes sacrificielles sont plus littéraires que religieuses, mais il reconnaît que pour atteindre son but horrifiant « the individual rubrics of the ceremony must somehow be drawn from the cultural inventory of religious ideas. Since the scene is a shocking one, the natural place to look for analogues is in the current prohibitions of religious groups... We might even chance to find somewhere a continuous series of prohibitions which Lollianos has inverted point by point, creating a sort of photographic negative » (p. 171). 
cannibale dans lequel des brigands mangent un enfant : la viande à

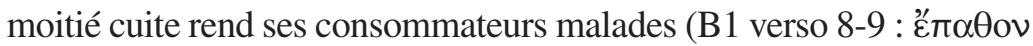

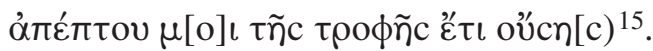

Ces trois textes de nature différente rendent compte de la présence de la tension entre le cru et le cuit dans les récits du mythe de Dionysos et dans la pratique rituelle liée à ce mythe, fût-elle réelle comme celle dont se fait l'écho le $P$. Gurôb, ou imaginaire comme celle décrite par Firmicus ou Lollianos. Ils renforcent l'hypothèse - déduite à partir de la rapidité avec laquelle Zeus fait son apparition dans les récits de Clément, Arnobe et Nonnos selon laquelle Dionysos n'était cuit qu'à moitié. Si cette hypothèse était avérée, ce détail mythologique serait une expression manifeste de la tension entre le cru et le cuit dans le mythe. On peut en approfondir la signification à trois niveaux d'interprétation, que nous qualifierons ici de structurel, narratif et anthropologique. Ces étiquettes, disons-le sans ambage, sont purement conventionelles et ne visent qu'à clarifier l'exposé.

15. Cette viande à moitié cuite pourrait être le cœur de la victime que les initiés mangent (B1, 12-13). Malheureusement il manque au texte environ

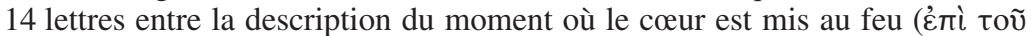

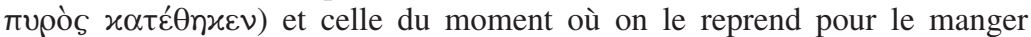

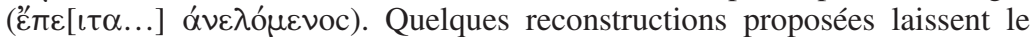

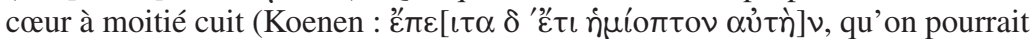

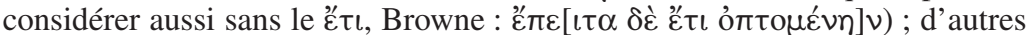

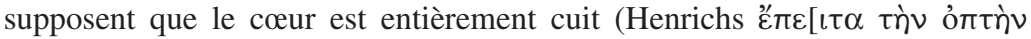

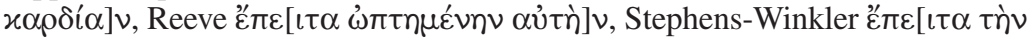

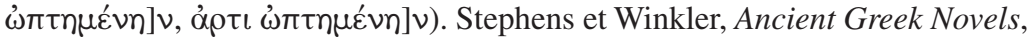
350 , allèguent que «we take Androtimos' complaint to be about the food they have eaten, which must have consisted of more than the heart, if it ever included it ». Mais c'est précisément l'importance donnée en B1 12-13 à la

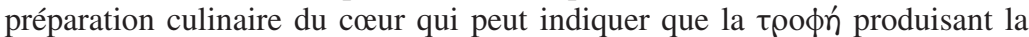
douleur est celle qui avait le plus de signification, c'est-à-dire, le cœur, dont ils

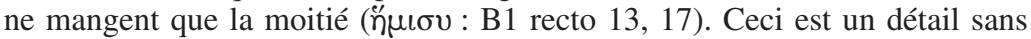
précédant qui rappelle l'idée d' " achèvement imparfait » qui semble présente dans ce genre de sacrifices sacrilèges comme signe de perversion rituelle (cf. point 4 infra). 


\section{OMOPHAGIE MÉNADIQUE ET SACRIFICE RITUEL}

Le premier niveau d'analyse, qualifié ici de structurel, est celui du rapport entre les traits les plus caractéristiques du ménadisme, le sparagmós et l'omophagie, et les éléments propres au sacrifice ritualisé, à savoir la coupure des chairs et leur mise au feu. Déchirer et manger une victime crue comme le font les ménades s'oppose évidemment en tant que débordement sauvage, à l'institution civilisée par excellence, le rituel du sacrifice ${ }^{16}$. L'épisode des Titans se situe à mi-chemin entre les deux pôles opposés, puisqu'il combine, d'un côté, les éléments ménadiques du diasparagmós (comme le montrent, par exemple, le verbe $\delta \iota \alpha \sigma \pi \alpha ́ \omega$ utilisé par Clément et la mention par Firmicus du déchirement d'un taureau vivant) et de l'enterrement des membres et, de l'autre, des éléments propres au sacrifice rituel dans lequel on cuit la victime, tels que le couteau, le feu, le tripode et la marmite. Dans les histoires de cannibalisme comme celles de Tantale, Thyeste ou Harpagos les éléments du sacrifice sont toujours présents et il n'y a aucune trace de diasparagmós et d'omophagie. Mais dans le mythe des Titans, les deux aspects sont mêlés et il serait erroné de vouloir les séparer dans un désir de clarté conceptuelle très rarement satisfait dans le domaine dionysiaque et spécialement dans les témoignages orphiques ${ }^{17}$. Aussi la demi-cuisson de Dionysos

16. Sur le sacrifice comme centre de la vie sociale grecque, $c f$. Jean Rudhardt: Notions fondamentales de la pensée religieuse et actes constitutifs du culte dans la Grèce classique, Paris, 1958. Sur l'omophagie ménadique comme transgression du sacrifice civique, $c f$. Dario Sabbatucci, Saggio sul misticismo greco, Rome, 1965, et Marcel Detienne, «Les chemins de la déviance : orphisme, dionysisme, pythagorisme », dans Orfismo in Magna Grecia. Atti del quattordicesimo Convegno di Studi sulla Magna Grecia, (Taranto, 6-10 ottobre 1974), Naples 1975, 49-79, et Id., Dionysos mis à mort, Paris, 1977.

17. Robertson, Orphic Myth, 222-226, essaie de séparer complètement les deux aspects du mythe : l'ancien mythe, dérivé du rituel delphique, raconterait le diasparagmós et l'enterrement de Dionysos, tandis que la cuisson de ses membres proviendrait de versions postérieures de poètes orphiques, qui auraient perdu le lien rituel avec le mythe primitif. Mais pour parvenir à une telle clarté, le savant américain doit émender l'un des témoignages les plus anciens, celui d'Euphorion (Schol. Lycophr. Al. $207=O F$ 36), selon lequel les Titans le mettent au feu. Selon Robertson (p. 225) le vers d'Euphorion serait : « comme s'ils le mettaient au feu ». Cela me paraît très peu vraisemblable. 
se présente-t-elle comme une tension entre les deux extrêmes, le cru et le cuit, qui maintient le mythe dans l'ambivalence et redouble ainsi son caractère unique : non seulement la victime est le dieu, mais le mythe lui-même ne saurait s'adapter complètement aux modèles "typiques" du meurtre dionysiaque ou du sacrifice cannibalesque.

\section{LA PERVERSION DU RITUEL SACRIFICIEL}

Le deuxième niveau d'interprétation, qu'on a baptisé ici de narratif, est lié à la considération du sacrifice titanique comme crime abominable. Pour l'auteur de l'épisode, très probablement un poète orphique ${ }^{18}$, l'impureté de ce sacrifice sacrilège, qui mérite le châtiment terrible de Zeus, se refléterait alors dans la perversion du processus rituel. L'importance spécifique des détails culinaires sacrificiels a surtout été soulignée par M. Detienne ${ }^{19}$, qui faisait remarquer que l'ordre bouillir-rôtir figurant dans les versions du mythe rapportées par Clément d'Alexandrie ${ }^{20}$ et les Problèmes pseudo-

$C f$. les éléments ménadiques en relation avec le mythe chez Diodore de Sicile 3.62.6, 5.75.4 ; Philodem. De Piet. 44. 16.1 Gomp.; Procl. In Plat. Crat. 406bc, In Alcib. 344, 31; In Tim 24e (I 173, 1 Diehl) 35b (II 198.2 Diehl) ; Olympiodore, In Plat. Phaedon. 61c, 67c ; Himer. Or. 9.4 ; Cornut. 62.10 ; Nonn. Abbas in Orat. contra Iul. 2.35 ; Lyd. De Mens. 4.51. Nous examinerons au point 5 des témoignages tardifs (Firmicus, un scholiaste de Clément, Photius), qui font du mythe un rituel purement ménadique.

18. La première mention faisant explicitement d'Orphée le narrateur du

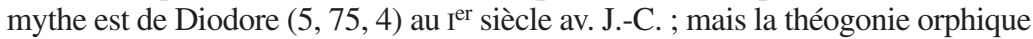
qui sert de base à la source de Clément remonte probablement au début de l'époque hellénistique ( $c f$. n. 9). Les allusions au mythe à l'époque classique (recueillies dans Bernabé, La toile...) ne mentionnent pas Orphée, mais il est probable qu'il ait déjà été le poète principal du mythe. On ne peut savoir si la théogonie partiellement conservée du Papyrus de Derveni faisait allusion à l'épisode.

19. Marcel Detienne, Dionysos mis à mort, 163-217 ; Marcel Detienne, Jean-Pierre Vernant (éds.), La cuisine du sacrifice en pays grec, Paris, 1979.

20. Protr. 18, 1, cité supra. Clément souligne la succession temporelle

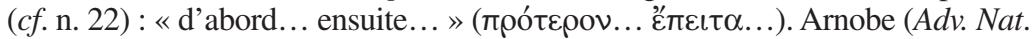
5,19 , cité supra), qui traduit le texte de Clément, ne la reprend pas. Firmicus Maternus confirme par une expression vague que le détail culinaire était significatif (De Err. 6, 3 : decocta variis generibus pueri membra consumunt). Si l'on 
aristoteliciens $^{21}$ est différent du processus sacrificiel habituel ${ }^{22}$. Si Dionysos était à demi cuit, ne serait-ce pas parce que ce détail contraste aussi avec la cuisson complète des victimes sacrificielles dans le sacrifice conventionnel ? La condition sine qua non d'une cuisson complète, et non à moitié, des viandes ressort clairement des formules homériques décrivant le sacrifice ${ }^{23}$. La nécessité de cuire complètement l'animal peut être motivée par la volonté d'assurer, d'une part, que les dieux reçoivent toute la fumée (knise) qui leur correspond et de marquer, d'autre part, clairement la frontière entre le cru et le cuit, qui sépare le civilisé du sauvage. Laisser la victime, dans ce cas Dionysos, à moitié cuit, empêche d'atteindre ces deux buts. La perversion d'un élément essentiel du processus sacrificiel pourrait être interprétée, au même titre que l'inversion de l'ordre rôtir-bouillir, comme un détail culinaire soulignant l'impiété du festin titanique ${ }^{24}$.

admet que les allusions homériques du texte de Clément (Il. 2.426-428, 4.49) procèdent du poème orphique servant de base à sa source $(c f$. mon article Fuentes cité en n. 9), alors le poète orphique aura peut-être joué sur les passages homériques décrivant la façon « orthodoxe » de cuisiner la viande, en s'y référant expressément pour souligner l' « hétérodoxie » dans le festin titanique.

21. Ps. Aristote Problemata 3, 43 : «Pourquoi n'est-il pas habituel (ou nomos) de rôtir le bouilli, alors que ce l'est de bouillir le rôti ? Est-ce en raison de ce qui est dit dans la téleté... ? » Detienne, Dionysos mis à mort, 173, traduit «n'est pas permis », mais ou nomos n'a pas la même force prohibitive que ou themis.

22. Cet ordre est différent, et non opposé au sacrifice normal. Robert Parker ("Early Orphism", dans A. Powell (éd.), The Greek World, Londres-New York, 1995, 509, n. 93) signale que la méthode des Titans ne constitue pas une inversion parfaitement symétrique de la norme sacrificielle (rôtir quelques portions, puis bouillir quelques autres). L'asymétrie rend moins persuasive l'interprétation anti-politique que Detienne (Dionysos mis à mort, 173ss et l'article cité en n. 16) donne à ce qu'il prend comme une inversion complète.

23. Il. $1.466=2.429$ : toutes les viandes ( $\pi \alpha ́ \nu \tau \alpha)$ doivent être soigneusement

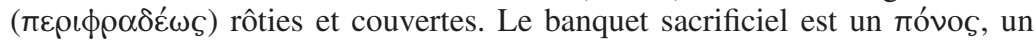
travail minutieux au terme duquel on peut commencer à fêter, mais pas avant. $C f$. Hdt. 1.119, où Astyage cuisine les membres du fils d'Harpagos jusqu'à ce

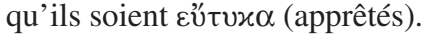

24. Detienne voit dans la perversion du rituel culinaire la proclamation d'un « chemin de déviance » qui aurait voulu rejeter avec ce mythe tout sacrifice comme crime d'anthropophagie. À mon avis, le mythe voulait signaler de cette façon l'impiété spécifique du sacrifice titanique, mais je doute qu'il contienne le rejet de tous les sacrifices sanglants, qui, précisément, suivraient l'ordre 
Toutefois, la signification originelle d'un détail narratif est très différente de celle qu'il peut avoir pour ses interprètes, et il ne faut pas imaginer que ce détail était présent ou revêtait une importance particulière dans toutes les versions du mythe : le mythe des Titans, notamment, comporte plusieurs détails (les éléments culinaires, le miroir, le plâtre, le cœur) potentiellement significatifs, qui demeurent inexploités ou sont développés de manière variable selon l'usage que l'on fait du mythe dans chaque cas particulier ${ }^{25}$. En fait, il semble que chaque usage du mythe soit différent selon le contexte dans lequel il intervient, et l'extension d'une interprétation particulière du récit à d'autres contextes est très problématique. Les significations potentielles de chaque détail n'étaient pas nécessairement prises en compte dans toutes les versions du mythe, puisqu'il n'y avait pas de « système doctrinal orphique » qui aurait imposé la transmission de tous les détails et, moins encore, de leur interprétation.

Ainsi, le fait que Dionysos soit à moitié cuit n'a eu d'importance, dans nos sources, que pour un poète orphique (et, peut-être, pour les érudits patréens désireux de donner une étymologie à un quartier de leur ville). Les deux premiers niveaux d'interprétation se référaient exclusivement à la possible signification du détail pour un narrateur du mythe ayant pu dire explicitement que les membres de Dionysos n'ont pas eu le temps de cuire complètement. Mais ce détail accidentel a cependant permis de focaliser un trait qui, lui, apparait dans toutes les versions du mythe : à savoir que les Titans n'achèvent pas

établi. En fait, le texte de Pausanias cité au début de cet article montre que le mythe était utilisé à Patras non pour rejeter la communauté politique, mais au contraire comme instrument d'identité de celle-ci et justification du culte publique de Dionysos Mésateus. Cf. Jan Bremmer, "Remember the Titans!", dans C. Auffahrt et L. Stuckenbruck (éds.), The Fall of the Angels, Leyde 2003, 53 : " surely an example of local appropriation of a Panhellenic myth ».

25. L'auteur des Problemata a prêté attention à l'ordre culinaire, parce qu'il voulait donner une justification religieuse - à côté de celle culturelle - à une coutume culinaire qui s'inscrit dans la question populaire des différences entre bouillir et rôtir (Aristote, Meteor. 4, 3, 380a36ss ; 381a27ss ; Probl. 5, 34, 884a36ss. Philocore, FGrH 328 F173 ; Plat. Rep. 372d-373a). Pour les possibles significations du plâtre utilisé par les Titans, $c f$. Pierre Ellinger, $L a$ légende nationale phocidienne, Paris 1993, 147-179 et Maurizio Bettini, « Un Dioniso di gesso », QUCC 43, 1993, 103-110. 
leur festin parce que Zeus entre en scène avant qu'ils puissent le faire. C'est autour de ce trait distinctif que s'articule le troisième niveau d'interprétation.

\section{LA THÉOPHAGIE SUPPRIMÉE}

Dans ce troisième niveau, que nous qualifierons d'anthropologique, on peut considérer que l'arrivée soudaine de Zeus, qui interrompt le festin titanique, s'inscrit dans une tendance généralisée à minimiser le rôle de Dionysos comme victime sacrificielle, même si on ne peut échapper au fait qu'il s'agit là précisément de l'événement central du mythe. Cela nous amène à proposer quelques réflexions sur la vexata quaestio de l'interprétation sacramentelle de la théophagie titanique.

Il faut d'abord rappeler que la majorité des témoignages qui rapportent cet épisode omettent de se référer à la manducation de Dionysos $^{26}$. Les allusions plus anciennes, bien que fragmentaires, de Callimaque et Euphorion (OF 34-36 B), ne parlent pas de l'ingestion de Dionysos. Proclus, dans plusieurs de ses allusions au mythe (recueillies dans $O F 210 \mathrm{~K}, 311 \mathrm{ss} \mathrm{B}$ ), évoque le démembrement de Dionysos et de son cœur, sauvé par Athéna, mais il ne laisse jamais entendre que les Titans l'auraient dévoré. Il est vrai que son exégèse néoplatonicienne ne requiert pas une telle référence, mais on retrouve la même absence d'allusions à la manducation dans les témoignages de Philodème, Diodore, Cornutus et Servius sur le démembrement et la résurrection de Dionysos (recueillies dans $O F 59 \mathrm{~B}$ ). C'est aussi le cas, on s'en souvient, de Nonnos de Panopolis. De son côté, Pausanias, on l'a vu, en parle de façon ambiguë : «dangers » $(7,18,4)$ et « souffrances » $(8,37,5)$. Enfin, le rhéteur Himérius, lorsqu'il se

26. Ce silence a des parallèles, p. ex. chez Euripide, Cyclope, 404-409 : de la cuisson des membres des compagnons d'Ulysse on passe directement à la sieste de Polyphème en omettant de décrire le moment même de l'anthropophagie ( $c f$. le même épisode décrit avec crudité en $O d$. 9.290-293). Mais chez les auteurs que nous citerons il ne s'agit pas d'une omission délicate de la description d'un fait sous-entendu, mais de l'absence totale de mention du fait lui-même ou de tout ce qui pourrait le suggérer. 
réfère à cet épisode dans l'un de ses discours $(9,45,4=302$ II, 318 VI, 324 III B), semble suggérer que Dionysos ne meurt même pas : il reçoit une blessure mortelle, ne peut plus se mouvoir puisque sa cheville est cassée, mais Zeus, «qui avait tout vu », le « réveille », puis chasse les Titans.

Ce silence des sources quant à l'ingestion de Dionysos par les Titans a été interprété par les « orphéo-sceptiques » plus radicaux ${ }^{27}$ comme une preuve de ce que les implications anthropologiques tirées du mythe (la nature à la fois dionysiaque et titanique des hommes issus de la fumée qui s'échappe des cendres des Titans) sont une construction tardive, puisque seul le néoplatonicien Olympiodore en donne la formulation expresse. Cependant, les allusions de Platon et d'autres témoignages anciens prouvent que l'interprétation anthropologique, pour laquelle l'ingestion de Dionysos est absolument indispensable, fait partie du mythe dès l'époque classique au moins ${ }^{28}$. À quoi doit-on, alors, une telle résistance à mentionner le festin des Titans ? Comme on l'a souvent proposé, l'horreur que cette suppression reflète est due à l'incompatibilité entre le fait qu'un dieu soit mangé et l'image publique du divin ${ }^{29}$. Ce que le détail narratif étudié ici dévoile est que cette suppression du tabou indicible - de l'arreton de la théophagie - peut être montrée non seulement à partir des textes qui omettent l'ingestion de Dionysos, mais aussi de ceux qui en font mention.

Outre les passages cités qui décrivent l'arrivée de Zeus, il n'y a que trois autres textes faisant allusion à la théophagie : Plutarque, Olympiodore et Firmicus Maternus. Laissant l'analyse de ce dernier pour plus tard, voyons comment les deux premiers la décrivent :

Plut. De esu carn. 1.7 996b : (Empédocle) parle dans ces vers allégoriquement des âmes, pour dire qu'elles sont enchaînées à des corps morts en châtiment pour meurtre, pour consommation de chair et pour cannibalisme ( $\left.\dot{\alpha} \lambda \lambda \eta \lambda{ }_{\text {o }} \phi \alpha \gamma^{\prime} \alpha_{S}\right)$. Pourtant il semble que cette doctrine soit encore plus ancienne, car les mythes qu'on raconte sur Dionysos qui fut déchiqueté et sur l'audace des Titans qui s'attaquèrent à lui, sur

27. Dernièrement L. Brisson et R. Edmonds, cités à la n. 5 .

28. Cf. Bernabé, La toile...

29. Walter Burkert, Greek Religion, Cambridge Mass., 1985, 64s ; Bernabé, La toile..., 432s. 
la punition de leur meurtre, c'est-à-dire sur leur foudroiement après qu'ils l'eurent goûté ( $\gamma \varepsilon v \sigma \alpha \mu \varepsilon \dot{\varepsilon} \nu \omega \nu)$, ceci est un mythe qui évoque par énigme la réincarnation. Car ce qu'il y a en nous d'irrationnel, de désordonné, de violent, ce qui n'est pas divin, mais démoniaque, les Anciens l'ont appelé “Titans" et cela parce que c'est ce qui a été puni et qui a reçu un châtiment (traduction adaptée à partir de Luc Brisson).

Olympiodor. in Plat. Phaedon. 1, 3 : On raconte que, par suite d'une machination ourdie par Héra, les Titans, ses gardiens, mirent en pièces

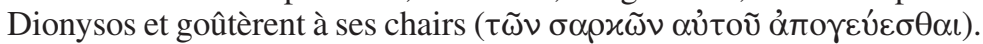
En colère contre eux, Zeus les frappa alors de sa foudre et le sublimé des vapeurs qui s'élevaient d'eux constitua une matière, dont vinrent les hommes. Il est donc interdit de se suicider, non parce que, comme semble dire le texte, nous sommes dans notre corps comme dans une prison, car cela est évident, et Socrate ne dirait pas qu'il s'agit là de quelque chose qui ressortit aux Mystères, mais parce que notre corps est «dionysiaque». En effet, nous sommes une partie de Dionysos, s'il est vrai que nous tirons notre origine du sublimé des Titans qui ont

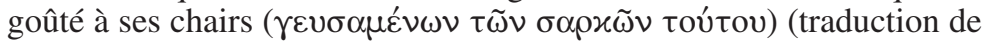
Luc Brisson, légèrement modifiée).

Rappelons-nous que Philodème, Diodore, Cornutus, Pausanias, Servius, Proclus et Nonnos ne mentionnaient pas du tout l'ingestion de Dionysos. Comme ils n'en avaient pas besoin pour leur usage ou interprétation du mythe, ils étaient heureux de l'omettre pudiquement. Himérius, de son côté, avant tout préoccupé de faire un discours agréable, manipule le mythe et évite d'évoquer la mort du dieu, en donnant au récit entier un ton christianisant, comme si tout faisait partie du plan de salut de Dieu le Père. Mais Plutarque et Olympiodore, comme on le voit dans ces textes, souhaitent tirer des conséquences anthropologiques du mythe : ils veulent que les hommes se composent des natures titanique et dionysiaque, et cela ne peut se faire que si les Titans ont mangé au moins une partie du corps de Dionysos. Par conséquent, ils essaient (et peut-être ne font-ils qu'imiter leur source, un poème orphique qui relatait l'épisode) de minimiser la théophagie, dont la mention est indispensable, par le verbe $\gamma \varepsilon v ́ o \mu \alpha \iota$ (goûter), désignant l'acte d'ingestion le plus minime qui soit, un verbe qui demande un génitif partitif impliquant plus de distance que l'accusatif. En revanche, quand Plutarque parle d'anthropophagie entre les hommes il n'hésite pas à utiliser la racine du

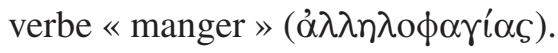


Leur attitude correspond à une tendance généralisée dans la mythographie grecque. La majorité des récits dans lesquels un enfant est dépecé et mangé dévoile la vérité à la fin du repas, une fois que l'enfant est complètement dévoré (p. ex. Harpagos, Thyeste, Térée). Mais ce sont des actes criminels commis par les hommes et l'horreur fait simplement partie du pathos de ces histoires. En revanche, quand les dieux sont les protagonistes du festin, tout change : aucune des sources rapportant le mythe de Lycaon ne nous permet de savoir si Zeus parvient à manger les chairs de son fils ${ }^{30}$. Ce silence ne s'explique que par l'horreur inspirée par l'idée qu'un dieu puisse participer au festin cannibale. C'est la même horreur que l'on trouve chez Pindare (Ol. 1.52: « je refuse !»), quand il raconte le mythe de Tantale, dans lequel les dieux se rendent compte juste assez tôt de la fraude pour éviter de manger les chairs de l'enfant Pélops, à l'exception de Déméter, qui en a donc "goûté » (Schol.

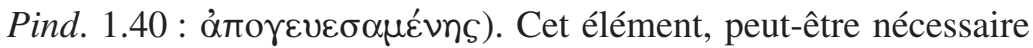
pour fournir l'étiologie de l'épaule d'ivoire de Pélops, concilie, dans la mesure du possible, la version la plus brute du mythe, dans laquelle l'enfant est mangé par les dieux ${ }^{31}$, avec les scrupules religieux que cela soulève. La même précaution qui apparaît lorsque les dieux mangent un enfant humain revient aussi, de manière encore plus marquée, quand c'est un dieu - Dionysos - qui est mangé : on s'efforcera alors de ne pas en faire mention, et si l'on ne peut éviter de le faire, on rendra l'épisode le plus court possible.

Ainsi, les Titans ne pourront faire plus que « goûter », non par des scrupules que leur nature brutale ne leur permettrait d'avoir, mais parce que Zeus arrive et les empêche de poursuivre. L'accélération de l'arrivée de Zeus devient alors la stratégie narrative des poètes orphiques (suivie fidèlement par leurs interprètes) pour adoucir un mythe théophagique dont le caractère permanent d'arreton fait

30. Apollod. Bibl. 3, 8, 1, Nik. Damasc., FGH 90 F 38. Sur tous ces mythes cannibalesques, $c f$. Walter Burkert, Homo Necans, Berkeley-Los AngelesLondres, 1983, 89-212.

31. Pind. Ol.1. 51 : фárov. Même si, comme le dit Gerber dans son commentaire ad loc, Pindare exagère avec des verbes au pluriel et que seule Déméter mange, la tension consistant à faire de tous les dieux des cannibales est toujours présente dans le mythe. 
que nous ne le connaissons que par des allusions. L'interruption du festin sacrilège permet d'omettre l'ingestion du dieu (et donne lieu à penser que ses membres n'ont pas eu le temps de cuire complètement), et, s'il est absolument nécessaire de mentionner la théophagie, on laissera juste le temps suffisant pour que les Titans y goûtent.

Il n'y a qu'une exception. Firmicus Maternus, qui raconte une version évhémériste du mythe selon laquelle Dionysos est le fils d'un roi de Crète, permet aux Titans de finir tranquillement leur festin, repoussant l'arrivée de Zeus au moment où le repas est achevé (De err. 6, 3) :

Là, on s'empare de lui, on le tue. Afin qu'aucune trace du meurtre ne puisse être retrouvée, la bande des gardes déchiquette les membres de l'enfant et s'en partage les débris. Alors, pour accumuler crime sur crime, et parce qu'on redoutait terriblement la cruauté du maître, ils cuisent de différentes manières les membres de l'enfant et les dévorent, se gavant d'un cadavre humain, chère inconnue jusqu'à ce jour (pueri membra consumunt, ut humani cadaveris, inauditis usque in illum diem epulis, vescerentur)... Une fois Jupiter revenu (reverso Iovi), sa fille lui rapporte le crime point par point (traduction de Robert Turcan, légèrement modifiée).

Dans son récit, les Titans mangent (consumunt, vescerentur) et ne se contentent pas de goûter à Dionysos. Mais cette exception confirme la règle qui vient d'être énoncée. Naturellement, une fois que l'histoire divine est traduite en termes humains, elle perd son caractère d'arreton : à partir du moment où l'on fait de Dionysos le fils d'un roi de Crète, il n'est plus un dieu, comme on le souligne en parlant de son «cadavre humain », et son sacrifice n'est pas pire que ceux des histoires de Thyeste ou Harpagos. Ainsi, pour les sources, vraisemblablement païennes, de Firmicus ${ }^{32}$, le festin d'un

32. Marek Winiarczyk, Evhemeri Messenii Reliquiae, Stuttgart-Leipzig, 1991, 55, suit l'opinion plus autorisée selon laquelle Évhémère ne peut être la source de Firmicus, comme on le pensait traditionnellement. Martin L. West, The Orphic Poems, Oxford 1983, 172 n. 101, reprend une observation orale de Burkert et compare Firmicus avec Sg. 14.15-16. Les deux textes parlent d'un roi qui, affligé par la mort de son fils, en fait une image qui deviendra objet de culte. Si l'on suppose que ces deux textes ont une source commune, on pourra faire remonter celle de Firmicus à l'époque hellénistique. 
enfant est un fait scandaleux et horrifiant, mais dès lors qu'il n'y a pas de théophagie, il n'est plus question de tabou.

On pourrait supposer qu'en tout état de cause un chrétien comme Firmicus n'avait aucune raison de respecter un tabou païen ${ }^{33}$. En principe, c'est vrai. Mais dans ce cas, comment peut-on expliquer que les apologistes chrétiens Clément et Arnobe omettent aussi le repas ? Comme on l'a vu, la description de l'acte d'ingestion manque dans leur version du mythe. Sans doute cette omission figurait-elle déjà dans la source de Clément, mais l'Alexandrin, et a fortiori son traducteur Arnobe, n'auraient pas renoncé à décrire ce détail s'ils l'avaient trouvé utile pour leur critique du mythe. Ce n'est assurément pas par piété païenne qu'ils l'omettent, mais parce qu'ils ont une raison apologétique de le faire : ils peuvent critiquer avec acharnement qu'on tue un enfant dans le mythe païen, mais ils ne peuvent s'en prendre au fait qu'on mange un dieu. Ce détail les conduirait inévitablement à soulever la question des différences avec la théophagie chrétienne dans l'Eucharistie, un débat qu'ils ont tout intérêt à éviter, puisque les chrétiens eux-mêmes étaient accusés de cannibalisme ${ }^{34}$. En revanche, la version évhémériste de Firmicus Maternus évite toute comparaison avec la théophagie eucharistique, et un chrétien comme lui peut donc raconter en détail cette histoire pour attaquer la religion grecque. Ainsi, l'évhémérisme fait du mythe un acte de cannibalisme pur et simple, facile à raconter, pour des raisons diverses, pour les païens comme pour les chrétiens.

L'attitude des chrétiens Clément et Arnobe devant le mythe des Titans aboutit ainsi à une suppression de la théophagie semblable à celle des auteurs païens devant le même mythe. Quoiqu'il se fonde sur des motifs fort différents, ce parallélisme révèle une perception, peut être en partie inconsciente, d'une certaine identité profonde entre la théophagie du mythe dionysiaque et celle pratiquée dans l'Eucharistie. Le païen Porphyre condamne lui aussi, dans un passage célèbre, l'Eucharistie comme anthropophagie répugnante, avec la

33. Comme le dit Chuvin (cf. n. 11).

34. Cf. Albert Henrichs, "Pagan Ritual and the Alleged Crimes of the Early Christians", dans P. Granfield et J. A. Jungmann (éds.), Kyriakon. Festschrift J. Quasten I, Münster, 1970, 18-35. 
même énergie qu'il attaque, en végétarien, le sacrifice sanglant ${ }^{35}$. Ces perceptions avaient-elles une raison d'être ? Je ne discuterai pas ici pour savoir si, dans l'Eucharistie, il y a, comme disent les modernes, une «sublimation» de l'instinct sacrificiel ${ }^{36}$. Prêtons plutôt attention aux possibles conséquences de cette équation sur l'autre pôle : peut-on aussi détecter la théophagie dans le sacrifice animal ? C'est ainsi qu'on arrive à une autre question classique, celle de l'interprétation sacramentelle du sacrifice grec ${ }^{37}$, à laquelle on se contentera de contribuer ici par quelques réflexions inspirées par les textes analysés. Mais auparavant faisons le point sur l'état de la question.

Il y a trois éléments en tension dans le sacrifice, l'homme, l'animal et le dieu, et il est tentant de vouloir arriver, par l'identification du dieu avec la victime que l'on mange, à une fusion complète des trois pôles, c'est-à-dire à l'union de l'homme avec la divinité par son ingestion sous la forme d'un animal. Mais cette tentation semble plutôt appartenir aux anthropologues modernes, comme projection plus ou moins consciente de l'Eucharistie chrétienne, qu'aux auteurs anciens, chez lesquels on ne trouve aucune trace d'une telle interprétation du sacrifice. Dans les rares récits de sacrifice humain,

35. Porphyre (fr. 69 Harnack) apud Macarius de Magnésie Monogénès 3.15 (c'est ainsi que Richard Goulet, dans son édition de 2003, a réintitulé l'œuvre traditionnellement connue sous le titre d'Apocriticon). L'attaque de Porphyre, qui se refère au passage de Jn 6,53, ainsi que la réponse de Macarius (3.23), sont très éclairants à propos des questions traitées ici.

36. C'est ainsi que le qualifie Albert Henrichs : "Human Sacrifice in Greek Religion: Three Case Studies", dans Le Sacrifice dans l'Antiquité, Entretiens Hardt 27, Vandœuvres-Genève 1981, 229-231. Il partage le point de vue de Charles Harold Dodd, The Interpretation of the Fourth Gospel, Cambridge 1953, 339. Cette identité anthropologique ne doit pas être confondue avec la dépendance de l'Eucharistie chrétienne à l'égard des cultes grecs, une idée en perte de vitesse aujourd'hui ; $c f$. Arthur Darby Nock, Early Gentile Christianity and its Hellenistic Background, New York-Londres, 1964, 68-86 et 109-147 ; Hans-Joseph Klauck, Herrenmahl und hellenistischer Kult, Münster, 1982.

37. $C f$. l'histoire du débat dans Dirk Obbink, "Dionysus Pored Out: Ancient and Modern Theories of Sacrifice and Cultural Formation", dans C. Pharaone et T. Carpenter (éds.), Masks of Dionysus, Ithaca 1994, 65-86. W. Burkert, Homo Necans, 75-78, 141. 
tel celui d'Iphigénie, l'homme peut prendre la place de la victime. Mais ce rôle est complètement impossible pour les dieux, toujours présents au sacrifice comme des hôtes ${ }^{38}$. C'est dans le domaine dionysiaque, où l'adhérent au culte (bacchos) arrive à l'identification avec le dieu (Bacchos), que l'on a mis tous les espoirs pour découvrir le sacramentalisme dans le monde grec ancien. Des auteurs très prestigieux et influents comme J. Harrison ou E. R. Dodds ont soutenu une conception du ménadisme encore très répandue, selon laquelle les ménades dévoraient le dieu Dionysos dans un rituel omophagique visant à entrer en pleine communion avec lui ${ }^{39}$. Leur idée semble aujourd'hui avoir perdu de sa popularité et la majorité des spécialistes de la religion grecque ont montré leur scepticisme ${ }^{40}$. Toutefois, leur

38. Plut. Suav. Viv. Epic. 21

39. Le point de départ est la théorie totémique du sacrifice sémite de William Robertson Smith, Lectures on the Religion of the Semites, Londres, 1894 ; James G. Frazer, The Golden Bough, Londres, $1912^{3}$; et surtout Jane Harrison, Prolegomena to the Study of Greek Religion, Cambridge, 1903 $\left(1922^{3}\right)$, 478-491, qui l'appliquent au monde grec et la popularisent. Otto Gruppe, Griechische Mythologie und Religionsgeschichte, Munich, 1906, 729-37 ; Lewis R. Farnell, Greek Hero cults and Ideas of Immortality, Oxford 1921, 161-168 ; Franz Cumont, Afterlife in Roman Paganism, Yale 1922, 120 ; Alfred Loisy, Mystères païens et mystère chrétien, Paris, 1930 ; Eric R. Dodds, The Greeks and the Irrational, Berkeley, 1951, 270-282 et dans son commentaire aux Bacchantes de 1960 (2 éd.), ainsi que Jan Kott, The Eating of the Gods. An Interpretation of Greek Tragedy, New York 1973, 186-230, sont quelques-uns des grands noms qui ont accepté cette théorie et dont l'influence est encore très grande. Hors du domaine de l'histoire religieuse ancienne, mais proche de toutes ces idées, on ne peut omettre le célèbre ouvrage de Sigmund Freud, Totem et Tabou, publié en 1912.

40. Cf. Albert Henrichs, "Changing Dionysian Identities", dans B. F. Meyer, E. P. Sanders (éds.), Jewish and Christian Self-Definition III, Londres, 1982, 159-160 ; Walter Burkert, Ancient Mystery Cults, Cambridge Mass, Londres, 1987, 110-112 ; Obbink, "Dionysus Pored Out", cité ci-dessus en n. 37. Déjà Ulrich von Wilamowitz-Moellendorff, Der Glaube der Hellenen, Berlin, 1931/2, II, 68 ; Walter F. Otto, Dionysos, Mythos und Kultus, Francfort-sur-le-Main, 1933, 99 et 121 ; et André Jean Festugière, « Les mystères de Dionysos », Revue biblique, 4 (1935), 366-396, considéraient l'omophagie comme la conséquence de l'exaltation dionysiaque, et non comme l'instrument pour l'atteindre. Le déclin de cette idée s'observe dans le revirement des auteurs qui l'ont défendue dans un premier moment pour la rejeter ensuite : Nock, Nilsson, Henrichs ( $c f$. les références dans Henrichs, "Changing Dionysian Identities", 235, n. 217). 
rejet n'est pas définitif ${ }^{41}$, sans doute parce qu'ils tiennent justement compte de l'indubitable sagesse des défenseurs du sacramentalisme et parce qu'il reste à expliquer certains témoignages dans lesquels cette théorie a pris racine. Il faudra alors se demander sur quelle base un tel édifice a été bâti.

En tant que tabou, la théophagie donne l'occasion de jouer avec elle comme d'une possibilité qui n'arrive pas à se réaliser. Il y a, en effet, quelques expressions ambiguës dans les Bacchantes d'Euripide, peut-être même dans sa mise en scène, et quelques représentations iconographiques du ménadisme, dans lesquelles on joue sur la possible identification de Dionysos avec la victime de la furie sauvage des ménades ${ }^{42}$. Mais cette identification ne devient pas explicite et ne dépasse en tout cas pas le niveau mythique, puisque les Bacchantes sont plutôt la représentation dramaturgique d'un mythe que le tableau d'un rituel existant au temps d'Euripide. Bien que le mythe rejoué par Euripide ait poursuivi l'imagination des anciens et des modernes comme reflétant une possibilité réelle d'expression de l'extase dionysiaque, les traces épigraphiques du ménadisme rituel sont très loin d'avoir un quelconque parfum eucharistique ${ }^{43}$.

Le seul mythe dans lequel on trouve explicitement la théophagie est celui des Titans. Le récit est précisément un arreton, parce qu'il

41. Henrichs, "Changing Dionysian Identities", 160, ne ferme pas totalement la porte : "While we cannot be sure that the Greeks identified Dionysus with their sacrifical animals, or that the historical maenads practiced omophagy in literal sense, the whole theory stays a splendid castle in the air. » Burkert, Ancient Mystery Cults, 111 : «Even if such scenes were more than phantasies, they did not amount to "eating the god". » Ces deux derniers, comme Obbink, «Dionysus Pored Out », reconnaissent qu' on peut parler de sacramentalisme pour le vin ( $c f$. n. 49 infra).

42. Cf. Burkert, Homo Necans, 75-78, 141. C'est à partir des expressions ambiguës des Bacchantes - où l'on suggère l'identification de Penthée avec une victime sacrificielle comme le taureau $(B a .742-745,1185)$, identifiée à son tour avec Dionysos ( $B a .920 \mathrm{~s})$ - que l'interprétation sacramentaliste du ménadisme (cf. note 39) s'est développée. L'identification de Penthée avec Dionysos était peut-être aussi soulignée par des artifices scéniques telle la perruque de Dionysos que Penthée finit par porter (cf. Kott, Eating of the Gods, 205-207).

43. $C f$. Albert Henrichs, "Greek Maenadism from Olympias to Messalina", HSCP 82 (1978), 149-152. 
réalise la possibilité de faire d'un dieu la victime sacrificielle, et ce dieu n'est autre que Dionysos. Mais on ne peut pas, une fois de plus, aller au-delà du niveau mythique, puisque, pour postuler l'ingestion sacramentelle de la chair du dieu dans un animal, il faudrait supposer que dans l'actualisation rituelle du mythe titanique, sur laquelle on a du reste très peu de témoignages ${ }^{44}$, on arrivait à l'identification de la victime sacrifiée avec Dionysos. Cependant, cette identification rituelle, qui aboutirait nécessairement à reconnaître la théophagie rituelle chez les Grecs, n'apparaît que dans trois témoignages chrétiens assez tardifs. Examinons-les :

Le scholiaste de Clément, vraisemblablement d'époque byzantine, procède à une fusion du mythe avec le rituel ménadique d'où il résulte un vague parfum eucharistique (Schol. Protr. 119.8.9) :

Partition impie des chairs : les initiés dans les mystères de Dionysos mangeaient la chair crue, célébrant cela comme signe $(\delta \varepsilon \tilde{\gamma} \gamma \mu \alpha)$ du dépècement que Dionysos a subi des mains des ménades.

De la même façon que le scholiaste semble croire que le rituel de la teleté était surtout de dévorer une victime qu'il considérait identifiée à Dionysos en commémoration de son sacrifice, un texte déjà mentionné de Firmicus Maternus $(E r r .6,5)$ fait aussi de la victime sacrificielle d'un rituel crétois la commémoration de Bacchos :

Ils rééditent point par point tout ce que l'enfant a fait ou souffert en mourant. Ils déchirent à belles dents un taureau vivant, en ranimant par des commémorations annuelles (annuis commemorationibus) leur goût des festins sauvages...

Finalement, on trouve dans le Lexicon du patriarche Photius (s. v. $\nu \varepsilon \beta \rho i ́ \zeta \varepsilon ı \nu)$ la même idée que laissent entrevoir le scholiaste de Clément et Firmicus :

«Faire le cerf » : porter une peau de cerf ou démembrer des cerfs :

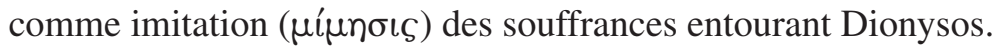

44. Le plus clair est le P. Gurôb ( $c f$. nn. 9 et 12). Les allusions aux dromena qui représentent le mythe des Titans dans des sources littéraires tardives, généralement néoplatoniciennes, semblent se référer à un rituel plutôt imaginaire que réel : p. ex. Macr. Somn. Scip. 1.12.12. La même chose vaut pour les dérivations littéraires du mythe comme le roman Phoinikika ( $c f . \mathrm{n} .14)$. 
Ces trois textes disent de manière explicite ce qu'aucun texte païen n'arrive à affirmer, à savoir que les rituels dionysiaques démembrent un animal en "commémoration" ( $\delta \varepsilon \tilde{\gamma} \mu \alpha \alpha$, commemoratio, $\mu$ í $\mu \eta \iota \varsigma)$ du démembrement de Dionysos. Certes, la commémoration n'est pas totalement synonyme d'identification de l'animal avec le dieu, mais elle est trop proche de la formulation de l'Eucharistie chrétienne ${ }^{45}$ pour ne pas penser à un glissement chez ces auteurs chrétiens vers une formulation quasi-eucharistique du sacrifice dionysiaque ${ }^{46}$. Le glissement s'opère facilement, puisqu'il suffit de pousser à l'extrême les éléments ménadiques du mythe des Titans : le scholiaste de Clément fait des ménades les belligérantes en lieu et place des Titans ; Firmicus attribue aux Crétois la prouesse de dévorer avec les dents seules un taureau vivant ; moins exagéré, Photius attribue aux célébrants des mystères dionysiaques un comportement ménadique. Naturellement, à part l'exagération de la présence d'éléments ménadiques dans le mythe des Titans, ces auteurs chrétiens ne mettent pas en doute qu'il s'agit d'un rituel effectivement réalisé, puisqu'ils sont prêts à attribuer toute sorte de crimes aux rituels extatiques païens. Mais leur valeur comme témoignage de la réalité rituelle est réduite, puisqu'il est clair qu'ils ne la connaissent pas du tout : on pourrait pardonner la confusion du scholiaste entre ménades et Titans ; mais on peut douter que les Crétois, pour forts et courageux qu'ils aient pu être, aient jamais essayé de dévorer un taureau vivant à belles dents.

Malgré leur faiblesse comme témoignages, ces trois textes ont été fondamentaux pour l'interprétation sacramentelle moderne du

45. La première mention de l'Eucharistie chrétienne, 1 Cor. 11.23-26, identifie déjà explicitement le pain et le vin avec le corps et le sang du Christ : «Ceci est mon corps... Cette coupe est la nouvelle alliance en mon sang. » Mais ce passage inclut aussi la commémoration comme clé interprétative du

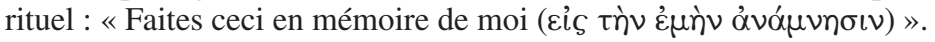

46. Les chrétiens ont également une position ambiguë par rapport à la viande des sacrifices païens, qu'ils refusent de manger. Quelques-uns supposent que les démons-idoles sont présents (littéralement, assis, $c f$. Robin Lane Fox, Pagans and Christians, Harmondsworth 1986, 444), quoiqu'ils ne les identifient jamais avec la victime sacrificielle. 
ménadisme grec, soutenue par Harrison et Dodds ${ }^{47}$. On voit donc que seuls les chrétiens ont dit explicitement ce que, comme les jeux verbaux d'Euripide ou le mythe des Titans le montrent, quelques Grecs ont été sur le point de dire, mais n'ont jamais voulu exprimer. Les Grecs n'ont jamais osé (du moins, nous n'en avons aucun témoignage) rendre explicite l'identité entre la victime et le dieu, ni proposer l'ingestion rituelle comme moyen pour arriver à une union avec la divinité. L'interprétation sacramentelle du sacrifice leur fait soutenir, suivant la tendance des sources chrétiennes, ce qu'ils n'ont jamais voulu formuler expressément. La raison de cette résistance est claire : l'horreur de la théophagie, une horreur qui se faisait évidente dans les versions du récit titanique qui voulaient même la passer sous silence comme événement mythique.

Toutefois, même si elle est erronée, la racine de l'interprétation eucharistique faite par les chrétiens et adoptée par quelques modernes peut nous éclairer : elle prend son origine dans la théophagie du mythe des Titans. Une certaine identité de fond avec l'Eucharistie chrétienne est évidente, comme on le voit dans le malaise des apologistes qui ont préféré omettre l'épisode. On peut donc achever cette étude par une réflexion générale qui découle naturellement des textes examinés : la théophagie des Titans est supprimée des textes grecs, alors que l'Eucharistie chrétienne ne l'est pas et, de plus, elle a été très rapidement acceptée chez les mêmes Grecs qui, quelques générations plus tôt, se gardaient de mentionner explicitement l'arreton de l'ingestion d'un dieu. Comment l'expliquer? C'est à mon avis le degré de symbolisation de l'Eucharistie qui permet d'éviter le tabou.

Le cas du vin peut aider à montrer plus clairement l'effet « détabouisant» de la symbolisation. Quoiqu'on puisse jouer sur l'identification de la victime sacrificielle avec le dieu, comme le fait Euripide, en se rapprochant même de la formulation verbale de cette identité, un dieu identifié à l'animal sacrifié et mangé heurte trop frontalement l'image fermement établie des dieux grecs. Par

47. Cf. Harrison, Prolegomena, 478-491; Dodds, Greeks and the Irrational, 277 n. 52. 
contre, les figures par lesquelles on donne à un aliment le nom d'un dieu sont très communes ${ }^{48}$. Elles paraissent avoir une valeur plutôt littéraire, comme le dit clairement Cicéron $(N D 3,41)$ : «Quand nous appelons les moissons Cérès, le vin Liber, nous employons bien sûr un vocabulaire courant, mais qui crois-tu assez fou pour considérer sa nourriture comme un dieu ? (trad. van den Bruwaene) ». Admettons que, comme le veut le pieux romain, il s'agisse toujours d'un usage rhétorique (même si son refus de considérer l'expression plus littéralement peut indiquer qu'il y avait des gens « assez fous » pour le faire). En tout cas, l'existence même de ce type d'expressions montre que le tabou consistant à identifier expressément le dieu avec un aliment ne fonctionne plus quand il ne s'agit pas d'un animal.

Il semble que même au niveau du rituel l'identification du vin avec le sang du dieu, Dionysos bien sûr, ait pu être formulée et acceptée parmi les Grecs ${ }^{49}$. Cela contraste avec l'absence d'identification du corps du dieu avec la victime sacrificielle, mais cette différence s'explique dans la mesure où, pour identifier le sang et le vin, on a besoin d'une symbolisation qui rend la théophagie moins immédiate et plus acceptable. L'Eucharistie chrétienne reprend une identification symbolique très répandue, celle du vin avec le sang, et elle y ajoute

48. Eur. Ba. 284 : « Ce dieu (scil. Bacchos), tout dieu qu'il est, coule en offrande aux dieux ». Timothée fr. 780 appelle le vin le « sang de Bacchos » ( $\alpha \tilde{i} \mu \alpha \beta \alpha x \chi i ́ o v)$ qui est mélangé «avec les larmes des nymphes », ce qui paraît être un usage surtout rhétorique ( $c f$. Hordern ad loc).

49. Il s'agit surtout des rituels où l'on boit le vin avec un ton sacrificiel comme dans les Anthestéries (Burkert, Homo Necans, 225). La même identification du vin avec la sang d'un dieu (Osiris) semble être présente dans les rituels transmis par quelques papyri magiques ( $c f$. Henrichs, "Human Sacrifice", 230, n. 3). Les mêmes auteurs ( $c f$. n. 40) qui rejettent le sacramentalisme pour le sacrifice animal l'acceptent pour le vin : Burkert, "Greek Mysteries", 111 ; Henrichs, "Changing Dionysian Identities", 160 ; Obbink, "Dionysos pored out", 70. Toutefois, il faut prêter attention à la remarque d'Obbink (p. 79) : « There was a consumption, rather than sacramental, ritual. » En effet, le mot « sacramental » transmet une théologisation étrange au sacrifice grec, de même qu'à la boisson rituelle du vin. Le concept de «transsubstantiation » n'existe pas dans le monde païen. 
une nouvelle, celle du pain avec le corps ${ }^{50}$. Ainsi, on peut penser que l'Eucharistie chrétienne en est venue à être facilement acceptée chez les Grecs, précisément grâce au haut degré de symbolisation atteint dans l'identification du corps du dieu sacrifié avec le pain, plutôt qu'avec une victime animale. Une telle symbolisation a rendu acceptable la formulation explicite, qui n'exige pas de suppression immédiate du sacrifice du dieu et de la théophagie. Qu'elle soit originellement une sublimation du sacrifice sanglant ou non, l'Eucharistie a effectivement atteint les mêmes résultats que si elle l'était.

\section{CONCLUSION : LES LUMIÈRES DES ERREURS}

Si l'on jette un regard rétrospectif sur l'enquête poursuivie, force est de constater un paradoxe dont il faut tenir compte dans l'étude des religions anciennes. Il y a toujours un grand nombre d'erreurs de perception sur celles-ci, dans les sources anciennes comme dans les études modernes, erreurs provenant d'intérêts idéologiques ou de projections souvent inconscientes. Mais ces erreurs, malgré la distorsion qu'elles font subir aux éléments particuliers comme au portrait général, peuvent jeter des lumières sur certains aspects qui sont laissés dans l'ombre par des approches plus objectives. Ainsi, on a commencé par la recherche d'une étymologie évidemment fausse qui nous a pourtant aidé à découvrir la trace d'un détail du mythe des Titans, la demi-cuisson de Dionysos, détail qui a permis d'approfondir de possibles éléments du mythe. Ainsi, le dépècement de Dionysos tient une position ambivalente entre le sacrifice ritualisé

50. La racine de l'identification du sang et du vin n'est pas nécessairement grecque : personnifier les raisins et faire du vin son « sang » est commun dans la Méditerranée, chez les Grecs comme chez les Hébreux ( $c f$. John P. Brown, Israel and Hellas I, Berlin-New York, 1995, 135). En revanche, pour l'identification du pain avec le corps il ne semble pas y avoir de parallèles clairs hors du christianisme (Henrichs, "Human Sacrifice", 230). L'apologiste Justin (Apol. 1, 54, 6, Dial. 69) accuse les mystères de Dionysos d'avoir copié le rituel eucharistique du vin ; il attaque aussi comme plagiat le rituel mithraïque du pain et de l'eau, qu'il sent trop proche de l'Eucharistie (Apol. 66), mais il s'agit probablement d'une similitude formelle. Aucune accusation similaire n'est jamais faite pour le sacrifice animal. 
et le diasparagmós ménadique, et il présente des traits sacrificiels étranges comme signe de perversion rituelle qui fait du sacrifice un meurtre punissable.

Mais surtout, l'examen des textes qui précipitent l'arrivée de Zeus pour ne pas permettre aux Titans d'achever (voire même de commencer) leur festin a conduit à découvrir que la présentation du mythe par les apologistes chrétiens, quoiqu'elle serve des intérêts complètement différents, révèle une réaction similaire à celle des auteurs païens qui, en principe, regardent le mythe de l'intérieur. Dans le récit du sacrifice titanique, que ce soit dans des sources chrétiennes comme dans des sources païennes, l'idée de manger la victime est toujours présente. Mais l'ingestion de Dionysos n'est mentionnée qu'autant qu'elle est absolument nécessaire pour l'interprétation que l'on veut donner du mythe. Le mythe des Titans a donc révélé une plasticité susceptible de multiples présentations. Pourtant, son élément constitutif, la théophagie, laisse toujours planer son ombre sur la narration.

Cet élément central du mythe a été le fondement de la transposition moderne des notions sacramentelles d'Eucharistie chrétienne dans le sacrifice dionysiaque grec. Cette interprétation erronée s'appuie sur des auteurs chrétiens tardifs qui ont projeté leurs conceptions sur une religion qu'ils méconnaissaient. Pourtant, les erreurs inconscientes se produisent quand le glissement s'opère facilement, et seules sont possibles les manipulations qui sont plausibles. La théophagie, toujours atténuée dans les textes grecs, cesse d'être un tabou indicible quand elle est présentée sous une forme symbolique : voilà pourquoi l'Eucharistie a triomphé là où on n'osait pas dire qu'un dieu est mangé. Les auteurs chrétiens tardifs, comme les modernes qui les ont suivis en faisant une lecture eucharistique du sacrifice, ont seulement considéré une certaine identité de fond, celle que donne l'idée implicite ou explicite de la théophagie, parce qu'ils n'ont pas prêté attention au pouvoir répressif du tabou. Comme les évhéméristes qui voient dans les mythes des récits historiques, seuls ceux qui ne prennent pas Dionysos pour un dieu osent dire : "il a été mangé ». L'Aussenperspektive, aussi éloignée qu'elle puisse être de la réalité, peut ainsi contribuer à comprendre plus profondément les informations transmises par l'Innenperspektive. 
Ainsi, certaines erreurs, même quand elles s'enchaînent les unes aux autres, se montrent quelquefois plus productives pour éclairer des zones d'ombre que les affirmations en tout genre et les preuves irréfutables, qui sont du reste assez rares dans le domaine des religions anciennes. Si les erreurs contenues dans cette étude peuvent avoir cette utilité, celle-ci n'aura pas été vaine ${ }^{51}$.

Miguel Herrero de Jáuregui

C/ Mayor 70

28013 Madrid (Espagne)

miguelhdj@yahoo.es

51. Je remercie A. Bernabé, W. Burkert, R. Gagné et J.-M. Roessli pour leurs remarques et suggestions, et tout spécialement ce dernier pour avoir rendu mon français plus lisible. 